\title{
Community Based Rehabilitation for Task shifting in Mental Health
}

\author{
Saroj Prasad Ojha $\cdot$ Sagun Ballav Pant
}

Published online: 29 November 2018

(C) Springer Nature India Private Limited 2018

The number of people affected by mental illness globally is growing, as humanitarian crises from conflict and natural disasters multiply-as seen in Syria, Yemen and Myanmar. Funding is scarce as mental health is rarely given precedence from both governments and donors. In LMICs, not only are there limited mental health services available, utilization of existing services is also poor for a multitude of reasons, including geographic, cultural, and financial access barriers. Integration of mental health services into primary care presents a strategic opportunity to overcome some of these access barriers and reach the largest number of people while minimizing stigma and discrimination [1].

The task shifting approach represents a low-cost solution to tackling gaps in health services in the developing world. WHO defines it as "the rational redistribution of tasks among health workforce teams" adding - in global guidelines issued in 2008-" specific tasks are moved, where appropriate, from highly qualified health workers to health workers with shorter training and fewer qualifications". A systematic review of task shifting found that it offers "highquality, cost-effective care to more patients than a

S. P. Ojha $(\bowtie) \cdot$ S. B. Pant

Department of Psychiatry and Mental Health, Institute of

Medicine, Tribhuvan University,

Maharajgunj, Kathmandu, Nepal

e-mail: ojhasp@yahoo.com physician-centred model". The study warned that the challenges are "adequate and sustainable training", but recommended that the technique should be considered for "careful implementation" where a shortage of healthcare human resources threatens programmes [2].

Care for most people with mental illness is best delivered in the community and evidence-based guidelines recommend combining both medication and a psychosocial intervention, such as communitybased rehabilitation (CBR). This is the most visible part of psychiatric care and as such represents the link to society. The attitude of the public towards mental health treatment is mostly influenced by what rehabilitation accomplishes or not. In fact, the US President's Freedom Commission on Mental Health 2002 declared that helping affected persons to achieve functional recovery is the main purpose of the mental health care system.

One of the important issues behind the CBR approach is 'coverage', the attempt to shift from 'everything for a few' to 'something for everyone'. This issue was perceived as important from the very beginning because it was estimated that in most developing countries, hardly $2-3 \%$ of those in need of rehabilitation services ever received them. The challenge that most developing countries face is to see how best to arrive at an optimum quality of services, given the limitations of providing a large coverage with limited resources. 
How the different domains of CBR can be implemented into available resources is well highlighted. These measures have been tried and tested. In 2004, a Mental Health Pilot Project was implemented in Gujarat State, India, for the rehabilitation of persons with mental disorders in community settings, along the lines of the CBR model [3]. Working closely with NGOs and local communities, this private-public partnership established referral links with government hospitals to provide medicines and counselling to affected persons. At the end of 18 months, 1206 people (diagnosed with schizophrenia, mania, depression and epilepsy) had accessed psychiatric services on a regular basis, and 272 people had recovered and resumed their earlier occupations. As a result of the pilot project, it may be concluded that rehabilitation of people with mental illness is possible outside of hospital settings.

With regard to concerns regarding service provision, critical interpretive review of the academic literature related to CBR in low- and middle-income countries and to indigenous communities in highincome countries identified five key topic areas related to ethical considerations [4]. The five key topic areas are partnerships among stakeholders, respect for culture and local experience, empowerment, accountability, and fairness in programme design. The eight ethical questions are linked to these topics and associated with how CBR practices reflect commitments to equity, respect, inclusion, participation, and social justice.

Although numerous benefits of CBR along with ways of tackling concerns are elucidated in the editorial, challenges still remain [5]. The development of a separate infrastructure only for CBR may be too costly and take long to take off. There is challenge in committing new resources into the community, coordinating and incorporating it into the existing community infrastructure and hence the inclusion of CBR into existing development structures. There are challenges to be overcome in sustainability of the program, inconsistencies of government policies collaboration, sharing and networking among various bodies charged with the responsibilities of providing CBR because of bureaucracy on the part of those involved in providing rehabilitation services.

Inadequate training of personnel in CBR provides the biggest challenge in providing family/communityoriented services. CBR content and methodology need to be strengthened in the education of all disciplines of extension workers. There is need for intensive advocacy and influencing of curricula development at the central levels and provision of training opportunities, for those working at the community levels.

\section{Bibliography}

1. Khasnabis C, Motsch KH, Achu K, Jubah KA, Brodtkorb S, Chervin P, et al. CBR and mental health [Internet]. World Health Organization; 2010 [cited 2018 Nov 7]. Available from: https://www.ncbi.nlm.nih.gov/books/NBK310918/.

2. Callaghan M, Ford N, Schneider H. A systematic review of task-shifting for HIV treatment and care in Africa. Hum Resour Health. 2010;8(1):8.

3. Joshi BR. CBR for inclusion of people with mental illness. Disabil CBR Incl Dev. 2013;24(3):123-30.

4. Clarke S, Barudin J, Hunt M. Ethics and community-based rehabilitation: eight ethical questions from a review of the literature. Physiother Can. 2016;68(2):197-205.

5. Samuel JU. Utilization of community based rehabilitation for persons' with disabilities (PWD) in Nigeria: the way forward. Eur Sci J ESJ [Internet]. 2015 Sep 28 [cited 2018 Nov 7];11(25). Available from: https://eujournal.org/index.php/ esj/article/view/6205. 